\title{
Measurement of Aerodynamic Sound Source around a Circular Cylinder by Particle Image Velocimetry*
}

\author{
Yasuyuki Oguma', Takayuki Yamagata2 ${ }^{2}$, Nobuyuki Fujisawa ${ }^{2}$ \\ ${ }^{1}$ Department of Mechanical Engineering, Nihon University, Koriyama, Japan \\ ${ }^{2}$ Visualization Research Center, Niigata University, Niigata, Japan \\ Email: oguma-y@mech.ce.nihon-u.ac.jp
}

Received 25 March 2014; revised 3 May 2014; accepted 12 May 2014

Copyright (C) 2014 by authors and Scientific Research Publishing Inc.

This work is licensed under the Creative Commons Attribution International License (CC BY).

http://creativecommons.org/licenses/by/4.0/

(c) (i) Open Access

\begin{abstract}
The purpose of this paper is to propose a sound localization method as an alternative of the time-resolved particle image velocimetry (PIV) system for detecting the aerodynamic sound source of a circular cylinder in a uniform flow. The sound source intensity of a circular cylinder in a uniform flow is evaluated by measuring the time-derivative of instantaneous velocity field in the flow field using a pair of planar PIV system. It allows the visualization of the sound source intensity distribution, which is the time-derivative of the vector product of vorticity and velocity. The experimental results indicate that the aerodynamic sound is generated from the separation point and the velocity fluctuation in the separating shear layer from the circular cylinder. These results agree qualitatively with the previous findings from experiment and numerical simulation, which supports the validity of the present experimental method for evaluating the sound source intensity distribution.
\end{abstract}

\section{Keywords}

Aerodynamic Sound, PIV, Noise Source, Circular Cylinder, Vortex Shedding, Experimental Technique

\section{Introduction}

The aerodynamic sound generated from a bluff body in a stream is an important topic of interest and has been studied in literature. A circular cylinder is a typical example of such a bluff body in a stream that generates 
aerodynamic sound, so that the mechanism of aerodynamic sound from the circular cylinder has been a topic of interests in literature [1]-[3]. Knowledge about the pressure distribution, fluid forces, flow separation, mean and turbulence characteristics on and around the circular cylinder is fundamental to understand the mechanism of aerodynamic sound. These analytical and experimental studies on the sound and the flow field are summarized in review papers [4] [5], while the numerical approach to the flow induced sound has been developed due to the recent advance of computational technology [6].

A variety of experimental methods for localizing the sound sources have been proposed in literature, such as the acoustic impedance method [7], microphone array method [8], cross-correlation method [9]-[11], and the method using time-resolved particle image velocimetry (PIV) [12] [13]. Among these experimental methods, the last two methods allow for localization of the aerodynamic sound sources because they can evaluate simultaneously the velocity field, which supports the understanding of the generation mechanism for aerodynamic sound. The cross-correlation method analyzes the relationship between velocity fluctuations in the near field and the sound pressure fluctuation in the far field of a circular cylinder. This method relies on the acoustic analogy that the sound source intensity is proportional to the vector product of vorticity and velocity in the flow field [10] [11]. The other approach is the evaluation of the cross-correlation between the fluctuating static pressure fluctuation and the sound pressure fluctuation emitted from a bluff body [11]. On the other hand, the method using time-resolved PIV provides the time-series data of instantaneous velocity field, which allows the evaluation of time-derivative of the vector product of vorticity and velocity in the flow field [12] and the evaluation of the pressure fluctuation on the cylinder surface [13]. This method using the time-resolved PIV is effective for evaluating the aerodynamic sound spectrum, although it is very expensive and requires too many PIV image data to analyze the time-series velocity data. Therefore, it is preferable to develop an alternative cost effective method.

The purpose of this paper is to describe a new experimental method focusing on the localization of the sound source distribution of a circular cylinder in a uniform flow. This method is based on the direct evaluation of the time-derivatives of the vector product of vorticity and velocity using a pair of standard PIV system.

\section{Theoretical Background}

According to the vortex sound theory [2] [3], the acoustic sound $p_{a}$ can be expressed by the following equation assuming that the flow is in low Mach number:

$$
p_{a}=\frac{\rho x_{i}}{4 \pi c r^{2}} \iiint \frac{\mathrm{d}}{\mathrm{d} t}(\boldsymbol{\omega} \times \mathbf{u}) \cdot G \mathrm{~d} V
$$

Here, $c$ : sound speed, $G$ : compact Green function, $r$ : distance from sound source, $t$ : time, $\boldsymbol{u}$ : velocity vector, $V$ : volume of interest, $x_{i}$ : position of interest, $\omega$ : vorticity vector. Note that the vector product of vorticity and velocity in Equation (1) is called as Lamb vector and the time-derivative of the Lamb vector in Equation (1) is the sound source intensity. Therefore, the time-derivative of the Lamb vector is a key parameter to be measured in the present study for localizing the aerodynamic sound source from a circular cylinder.

\section{Experimental Method}

\subsection{Experimental Apparatus}

The experiment was carried out in an acoustic open-jet wind tunnel, which has been described in Ref. [14]. The test section has a dimension $190 \times 190 \mathrm{~mm}$ with $600 \mathrm{~mm}$ long. A circular cylinder of $15 \mathrm{~mm}$ in diameter was placed horizontally $300 \mathrm{~mm}$ from the nozzle exit and at mid-height of the wind tunnel. The test circular cylinder was made of acrylic resin material and supported by two vertical side plates made of transparent acrylic resin. The flow around the circular cylinder in a uniform flow was observed through the side plates. The top and bottom of the test section were open to the atmosphere. The experiment was carried out in a mean velocity of $U_{0}=30$ $\mathrm{m} / \mathrm{s}$, which corresponds to a Reynolds number $\operatorname{Re}\left(=U_{0} d / v\right)=3 \times 10^{4}$, where $v$ is the kinematic viscosity of working fluid of air. Note that the mean velocity in the test section was uniform within an accuracy of $\pm 1 \%$, and the free-stream turbulence level was about $1 \%$ [14].

\subsection{Measurement of Sound Source Intensity}

In order to evaluate the sound source intensity in the flow field, a pair of standard PIV system is introduced into 
the measurement. Each PIV system consists of a CCD camera $(1280 \times 1024$ pixels with 12 bits $)$, Nd:YAG lasers (50 mJ/pulse with 30 pulses/sec) and a pulse controller. An illustration of the experimental system is described in Figure 1. It should be mentioned that this experimental system allows the measurement of instantaneous planar velocity field in a short time difference, which results in the evaluation of time derivative of the sound source intensity in the plane of measurement. The principle of the measurement is that the camera A captures the smoke image illuminated by the 1st and the 2nd laser shots and the camera B captures the smoke images by the 3rd and 4th laser shots. Then, two pairs of images are analyzed by the cross-correlation analysis with sub-pixel interpolation technique to obtain the velocity vectors around the circular cylinder in a short time difference. The time derivative of the velocity vectors is calculated from the two velocity vectors at the same position of the flow field. It should be mentioned that the time difference between the 1st and 2nd images is set to $5 \mu \mathrm{sec}$, which is true for the 3rd and 4th images. On the other hand, the time difference between the 1st and 3rd images, which corresponds to the time difference between the two instantaneous velocity vectors, is set to $200 \mu \mathrm{sec}$. This time difference is determined from the consideration of pixel resolution of the velocity measurement, which is about 0.1 pixels in the present PIV analysis, while it should be much smaller than the time interval of vortex shedding $2.7 \mathrm{msec}$. It should be mentioned that this PIV system allows the flexible setting of the time difference between the PIV images and the cost is an order of 1/10 in comparison with the time-resolved PIV system.

\section{Results and Discussion}

\subsection{Sound Spectrum}

The sound spectrum generated from a circular cylinder was measured by a microphone of a diameter of 12.7 $\mathrm{mm}$, which was located on the top side of the circular cylinder with a distance of $500 \mathrm{~mm}$. The experimental result is shown in Figure 2, which shows the sound spectrum with and without the circular cylinder in a uniform flow. It is found that the sound spectrum with the circular cylinder has a peak around $370 \mathrm{~Hz}$ due to the generation of vortex shedding from the circular cylinder. The Strouhal number $\operatorname{St}\left(=f d / U_{0}\right)$ is evaluated as 0.19 , which is in close agreement with that of the vortex shedding from a circular cylinder in literature [5].

\subsection{Localization of Aerodynamic Sound Source}

Figure 3 shows an example of instantaneous velocity and vorticity field around a circular cylinder Figure 3(a) and the vector product of vorticity and velocity Figure 3(b), which are obtained from the smoke images taken by camera A. Note that the vorticity is evaluated from the circulation method [15]. These results show a high intensity of vorticity near the separation point and along the shear layer. The instantaneous velocity field around the circular cylinder after $200 \mu \mathrm{sec}$ is similarly obtained from the smoke images taken by the camera B. Then, the instantaneous sound source intensity is evaluated as shown in Figure 3(c) by evaluating the time derivative of the Lamb vector. The uncertainty in the time-derivative of the Lamb vector is about $10 \%$. The instantaneous sound source intensity distribution shows a high intensity along the shear layer originating from the point of separation and in the wake of the circular cylinder. Thus, the velocity and vorticity fluctuations in these regions could contribute to the generation of aerodynamic sound from the circular cylinder.

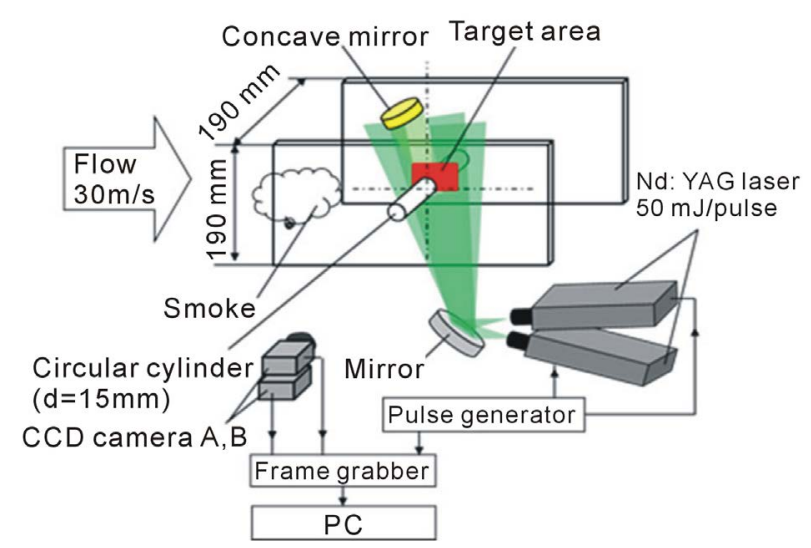

Figure 1. Experimental setup and measuring system. 


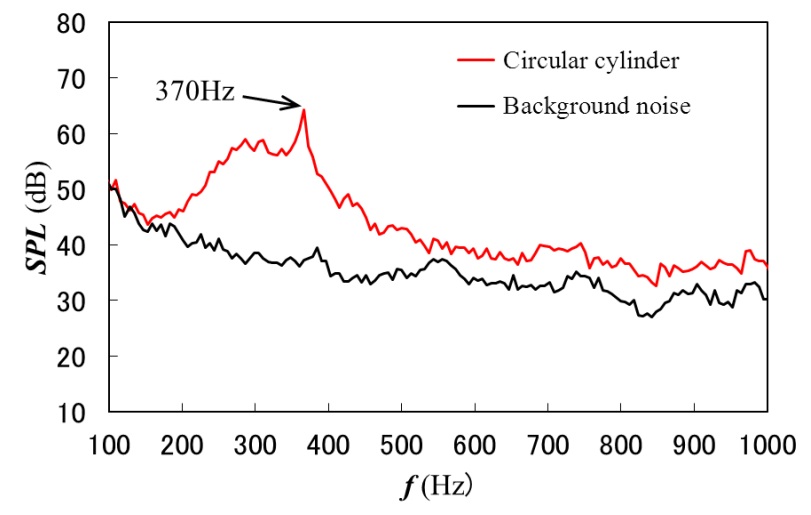

Figure 2. Frequency spectrum of aerodynamic sound.
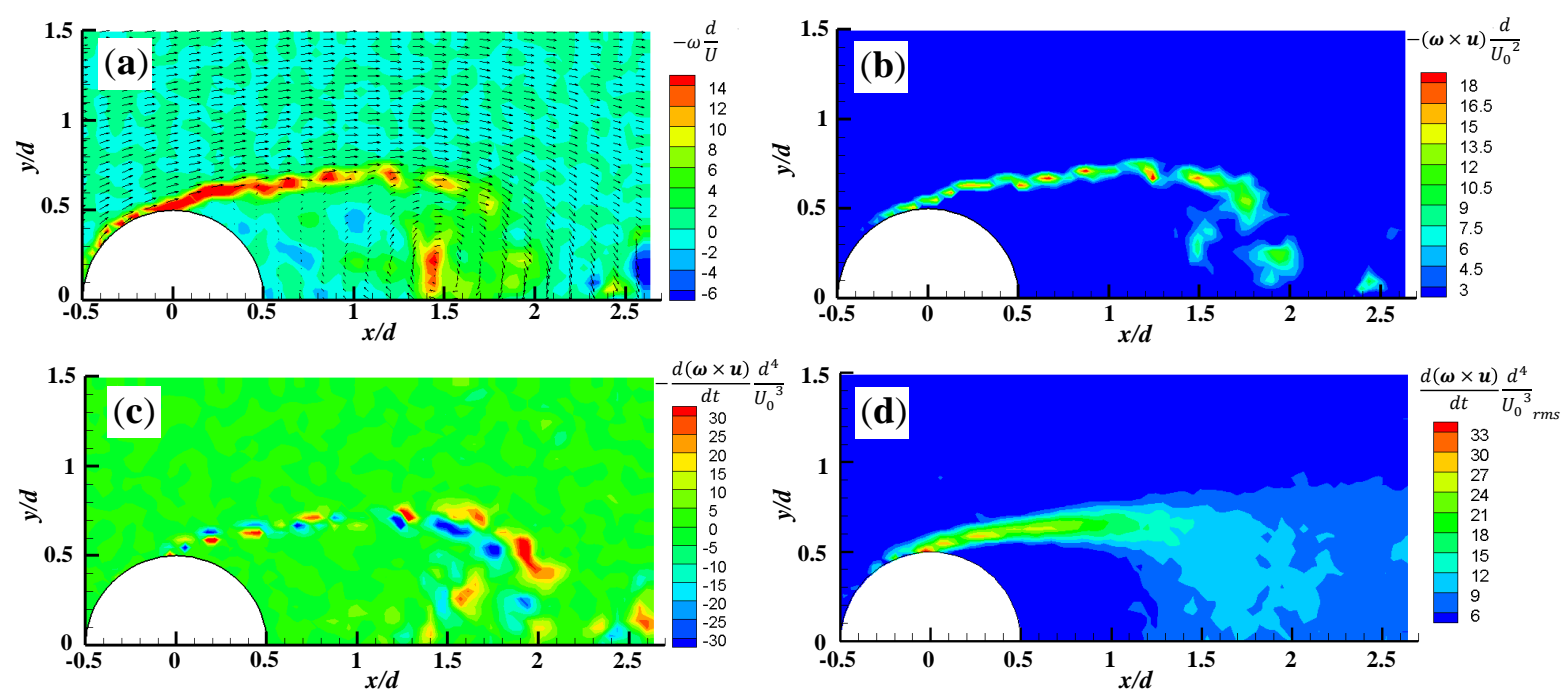

Figure 3. Examples of instantaneous flow and sound source intensity: (a) Instantaneous distribution of velocity and vorticity; (b) Instantaneous distribution of Lamb vector; (c) Time-derivative of instantaneous Lamb vector; (d) RMS of sound source intensity distribution.

In order to visualize the location of the sound source distribution, the RMS sound intensity is evaluated from 600 instantaneous velocity fields taken at $4 \mathrm{~Hz}$ in the flow around the circular cylinder. Figure 3(d) shows the RMS sound source intensity distribution in the whole area of interests of the circular cylinder. It is seen that the highest sound source intensity appears near the separation point and along the shear layer, which is similar to the instantaneous sound source distribution in Figure 3(c). However, the sound source intensity in the wake of the circular cylinder does not show high intensity in the wake and it is distributing broadly, reflecting the dynamic nature of vortex shedding. It should be mentioned that the present results agree qualitatively with the experimental and numerical results of aerodynamic sound source for a circular cylinder in literature [4] [5] [11], which suggests the reliability of the present experimental method for localizing the sound source distribution. The quantitative evaluation of sound intensity from the PIV measurement is the next step of research.

\section{Conclusion}

The measurement of sound source intensity distribution around a circular cylinder is carried out using a pair of PIV system as an alternative of the time-resolved one. This system allows the measurement of sound source intensity, which is the time derivative of the vector product of vorticity and velocity vector. The experimental result indicates that the large magnitude of sound source intensity is observed from the shear layer originating from the separation point and in the separating shear layer. This result agrees with the previous experimental and 
numerical observations of sound source distribution, which supports the usefulness of the present system.

\section{Acknowledgements}

The authors thanks to Dr. A. Nashimoto and Dr. T. Nakano from Mitsuba Corporation for their helpful discussion during the course of this experiment.

\section{References}

[1] Lighthill, M.J. (1952) On Sound Generated Aerodynamically: General Theory. Proceedings of the Royal Society of London A, 211, 564-587. http://dx.doi.org/10.1098/rspa.1952.0060

[2] Powell, A. (1964) Theory of Vortex Sound. Journal of Acoustical Society of America, 36, 177-195. http://dx.doi.org/10.1121/1.1918931

[3] Howe, M.S. (2002) Theory of Vortex Sound. Cambridge University Press, Cambridge. http://dx.doi.org/10.1017/CBO9780511755491

[4] Blake, W.K. (1986) Mechanics of Flow-Induced Sound and Vibration, Vol. 1, General Concepts and Elementary Sources. Academic Press, Waltham, 219-287.

[5] Norberg, C. (2003) Fluctuating Lift on a Circular Cylinder; Review and New Measurements. Journal of Fluids and Structures, 17, 57-96. http://dx.doi.org/10.1016/S0889-9746(02)00099-3

[6] Daude, F., Berland, J., Emmert, T., Lafon, P., Crouzet, F. and Bailly, C. (2012) A High-Order Finite-Difference Algorithm for Direct Computation of Aerodynamic Sound. Computer and Fluids, 61, 45-63. http://dx.doi.org/10.1016/j.compfluid.2011.08.017

[7] Jones, M.G. and Stiede, P.E. (1997) Comparison of Methods for Determining Specific Acoustic Impedance. Journal of the Acoustical Society of America, 101, 2694-2704. http://dx.doi.org/10.1121/1.418558

[8] Bersikow, B. (1996) Experiences with Various Configurations of Microphone Arrays Used to Locate Sound Sources on Railway Trains Operated by the DB AG. Journal of Sound and Vibration, 193, 283-293. http://dx.doi.org/10.1006/jsvi.1996.0269

[9] Nakano, T., Fujisawa, N. and Lee, S. (2006) Measurement of Total-Noise Characteristics and Periodic Flow Structure around NACA0018 Airfoil. Experiments in Fluids, 40, 482-490. http://dx.doi.org/10.1007/s00348-005-0089-2

[10] Henning, A., Kaepernick, K., Ehrenfried, K., Koop, L. and Dillmann, A. (2008) Investigation of Aeroacoustic Noise Generation by Simultaneous Particle Image Velocimetry and Microphone Measurements. Experiments in Fluids, 45, 1073-1085. http://dx.doi.org/10.1007/s00348-008-0528-y

[11] Oguma, Y., Yamagata, T. and Fujisawa, N. (2013) Measurement of Sound Source Distribution around a Circular Cylinder in a Uniform Flow by Combined Particle Image Velocimetry and Microphone Technique. Journal of Wind Engineering and Industrial Aerodynamics, 118, 1-11. http://dx.doi.org/10.1016/j.jweia.2013.04.003

[12] Schroeder, A., Dierksheide, U., Wolf, J., Herr, M. and Kompenhans, J. (2004) Investigation of Trailing-Edge Noise Sources by Means of High-Speed PIV. 12th International Symposium on Applications of Laser Techniques to Fluid Mechanics, Lisbon, 12-15 July 2004.

[13] Moore, P., Lorenzoni, V. and Scarano, F. (2011) Two Techniques for PIV-Based Aeroacoustic Prediction and Their Application to a Rod-Airfoil Experiment. Experiments in Fluids, 50, 877-885. http://dx.doi.org/10.1007/s00348-010-0932-y

[14] Nakano, T., Fujisawa, N., Oguma, Y., Takagi, Y. and Lee, S. (2007) Experimental Study on Flow and Noise Characteristics of NACA0018 Airfoil. Journal of Wind Engineering and Industrial Aerodynamics, 95, 511-531. http://dx.doi.org/10.1016/j.jweia.2006.11.002

[15] Raffel, M., Willert, C.E. and Kompenhans, J. (1998) Particle Image Velocimetry, a Practical Guide. Springer, Berlin, 147-171. http://dx.doi.org/10.1007/978-3-662-03637-2_6 
Scientific Research Publishing (SCIRP) is one of the largest Open Access journal publishers. It is currently publishing more than 200 open access, online, peer-reviewed journals covering a wide range of academic disciplines. SCIRP serves the worldwide academic communities and contributes to the progress and application of science with its publication.

Other selected journals from SCIRP are listed as below. Submit your manuscript to us via either submit@scirp.org or Online Submission Portal.
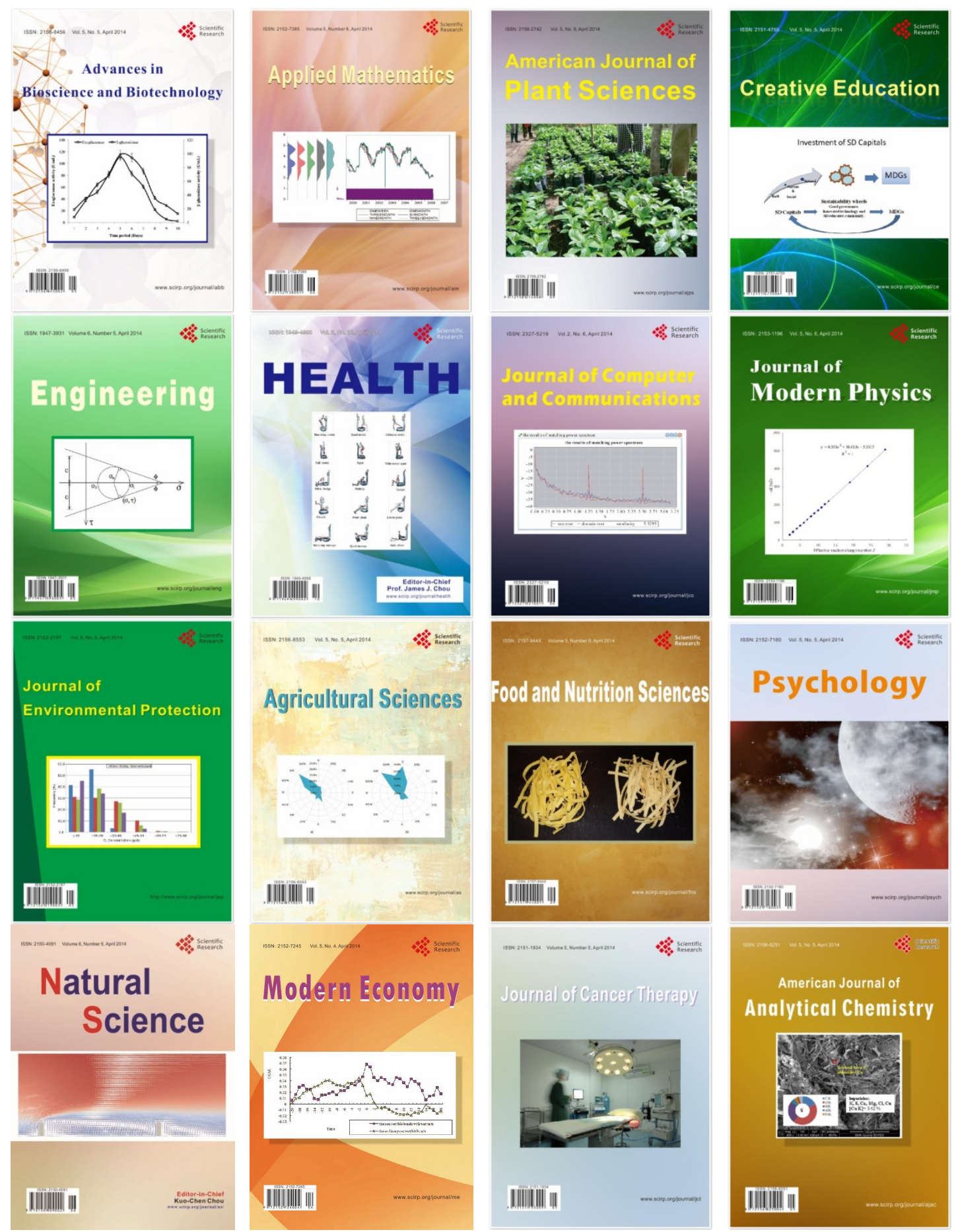